CELL COMMUNICATION Cells Swapping RNA

from our Cell Biology Correspondent THE notion that cells, when in contact, exchange nucleic acids and other macromolecules has never lacked enthusiastic advocates and has appeared in many guises, the most recent being Temin's protovirus theory. But. to date, the only substantiated examples of intercellular chemical communication involve the passage of small molecules such as nucleotides-a phenomenon which Subak-Sharpe and his colleagues have aptly termed metabolic cooperation-and ions. There is, of course, ample circumstantial evidence to hint that the exchange of macromolecules also probably occurs but no conclusive and direct experimental evidence. The reason is obvious enough. In order to demonstrate the swapping of macromolecules between cells the experimenter has to be able to distinguish donor from recipient cells : he must be able to separate them and he must, above all, prove that the molecules being shunted around are indeed macromolecules rather than their low molecular weight precursors. These are difficult criteria to meet but not, it might be thought, insuperably difficult given ingenuity and patience.

Kolodny (Exp. Cell. Res., 65, 313 : 1971) clearly shared this view; to achieve the separation of donor and recipient cells, he has exploited the fact that mouse $3 \mathrm{~T} 3$ fibroblasts ingest tantalum, without apparent adverse effects, and as a result become considerably denser. In brief, he splits a culture of mouse 3T3 cells and allows one half to ingest tantalum and at the same time incorporate ${ }^{3} \mathrm{H}$ or ${ }^{14} \mathrm{C}$-uridine of ${ }^{3} \mathrm{H}$-methylmethionine. He then mixes and cocultivates these cells with the other half of the original population exposed neither to tantalum nor labelled NHA precursors. After varying periods of cocultivation the tantalum containing cells can be separated from the others by centrifugation through a Ficoll gradient. It then becomes a simple matter of assaying for radioactive RNA in the separated populations of cells.

In such experiments after cocultivation lasting 1 to 4 hours the recipient cells, those without tantalum, have 10 per cent of the TCA precipitable counts found in the donor, tantalum containing cells, and after 18-20 hours the recipients contain 23 per cent of the radioactivity of the donor cells. Granting that Kolodny's separation procedure is really as efficient as he claims, these results must mean that either labelled RNAs or labelled nucleotides are being transferred from cell to cell : cell fusion as a cause of the transfer can be ruled out.

Unfortunately, Kolodny's evidence that it is indeed high molecular weight RNA that is transferred is indirect. For example, he shows that exposing the cells to actinomycin during cocultivation, at doses which inhibit RNA synthesis by 97 per cent, does not suppress transfer; he finds that the RNA precursor pools in both recipient and donor cells after cocultivation are not labelled above background; and he finds that thymidine incorporated into donor cell DNA does not find its way into recipients although ${ }^{3} \mathrm{H}$-methyl groups incorporated from ${ }^{3} \mathrm{H}$-methyl-methionine into donor RNA are transferred. Finally, Kolodny has shown that labelled ribosomal and transfer RNAs can be extracted from recipient cells after cocultivation.

Of course all these lines of evidence provide compelling but not conclusive support for the contention that intact RNA is transferred. To clinch the argument Kolodny must either show the transfer of a genetically marked RNA that could only come from the donor cells or else show that a physi- cal barrier between donors and recipients, which is permeable to nucleotides but impermeable to RNA, blocks the transfer. The last has clearly not been heard of this fascinating story, and in his concluding remarks Kolodny hints of things to come by saying "preliminary experiments with SV40 transformed 3T3 cells using techniques described indicate a lack of RNA transfer between these cells".

\section{SPORTFISH \\ Billfish Biology}

from our Marine Vertebrate Correspondent

IT is a paradox of marine biology that some of the least known fishes are the largest. Among the groups of which this is especially true are the billfishes of the family Istiophoridae, which include several well known sportfish such as the sailfish, the marlins, and spearfishes of tropical and subtropical oceans. Although these animals have

\title{
Catenated R Factor DNA from Minicells
}

THERE are numerous technical difficulties in the study of bacterial plasmids, not the least of which is the purification of their DNA. The DNA of antibiotic resistance factors $(R)$ can be readily separated from the chromosomal DNA of Proteus mirabilis by buoyant density centrifugation but not from that of Escherichia coli, but as some $\mathbf{R}$ factors show different properties of stability and replication in these two hosts (Cohen et al., J. Mol. Biol., 50, 671: 1970 ) it is of interest to be able to prepare the DNA from each.

The two standard techniques for separating $\mathrm{R}$ factor and $E$. coli DNA are caesium chloride-ethidium bromide centrifugation and bulk nitrocellulose adsorption. These methods both rely on the fact that $\mathrm{R}$ factor DNA exists as covalently closed circles and so they are unlikely to yield any other molecular forms of DNA. In next Wednesday`s Nature New Biology, Cohen et al. describe a technique designed to avoid this bias. They utilize the observation reported last year by Roozen et al. (Genetics, 64, 554 ; 1970) and Levy (Nature, 227, $606 ; 1970$ ) that $\mathrm{R}^{\perp}$ derivatives of a minicell-producing strain will segregate $R$ factor DNA, but not chromosomal DNA, into their daughter minicells, giving a biological enrichment for $\mathrm{R}$ factor DNA.

Cohen et al. have isolated DNA from $\mathrm{R}^{-}$minicells and have analysed it by centrifugation in caesium chloride with ethidium bromide. This has revealed three peaks of DNA; one with a density characteristic of covalently closed circular DNA, another with that of open circular DNA and an unexpected third peak with an intermediate density suggestive of catenated $\mathbf{R}$ factor DNA. This classification is supported by the sedimentation pattern of the DNA on sucrose gradients, and by examination of the peaks with an electron microscope. The distribution of the $R$ factor DNA between the three peaks varied from experiment to experiment, as did the proportion of the molecular forms within each peak, but in each case the densest peak contained predominantly supercoiled DNA, the intermediate peak contained singly or doubly open catenanes and the lightest peak contained open monomers.

Catenated DNA has been observed in mitochondrial DNA from several sources, and in Salmonella infected with P22, but in each case its origin is something of a mystery. Hudson et al. (Cold Spring Harbor Symp. Quant. Biol., 33, 435 ; 1968) suggest that they could arise from recombination or replication of circular DNA, or from an equilibrium between linear and circular forms. None of these explanations. however, is entirely satisfactory.

It is probably premature at this stage to speculate as to their origin from $R$ factors for, as Cohen et al. point out, it is not yet clear whether or not they are just a peculiarity of minicell producing strains or of the minicells themselves. Should they prove to be of biological significance and not just the byproducts of recombination, then they may shed a little light on the somewhat obscure question of the form in which plasmids exist within the cell. 